\title{
QUALIDADE DE DADOS DOS SISTEMAS DE INFORMAÇÃO DO DATASUS: Análise crítica da literatura
}

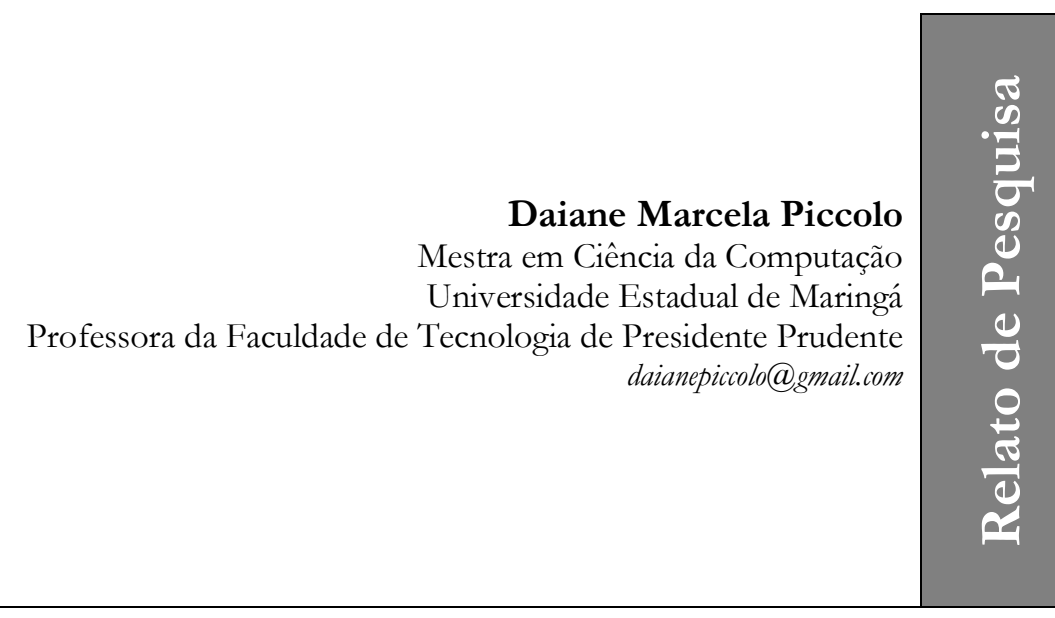

\section{Resumo}

Obter dados precisos com garantia de qualidade é um fator primordial para análises e melhores tomadas de decisões, em todos os campos. A área da saúde disponibiliza dados por meio do sistema do DATASUS, que integra diversos sistemas relacionados a questões de saúde. Entretanto, a garantia da qualidade dos dados é condição essencial para a análise objetiva da situação sanitária, assim como para a tomada de decisões e para a programação de ações de saúde. O trabalho constitui-se de uma pesquisa exploratória com abordagem qualitativa e quantitativa, cujo objetivo é identificar e analisar estudos que têm abordado questões de qualidade em sistemas de informação do DATASUS, no qual se obteve um total de sete artigos selecionados que abordaram questões de qualidade. Entretanto, notou-se que ainda há a necessidade de desenvolver novos estudos que tenham como objetivo avaliar a qualidade dos sistemas do DATASUS, como estratégia de melhoria para melhor utilizá-los.

\section{Palavras-chave}

Estatísticas vitais. Sistemas de informação. Qualidade.

\section{INTRODUÇÃO}

Com o crescimento dos dados ao longo dos anos, é perceptível o investimento de empresas em tecnologia que proporcionam integração das informações. Para tanto, os sistemas de informação tornaram-se instrumentos indispensáveis, uma vez que são responsáveis pela coleta, processamento, armazenamento e distribuição de informações para análises e melhor tomada de decisão em organizações (O'BRIEN et al., 1995). Entretanto, é importante levar em consideração a qualidade das informações, ou seja, garantir que os dados depois de armazenados sejam disponibilizados de forma estruturada com garantia de qualidade, para que empresas e usuários consigam ter resultados mais precisos em suas análises, o que na opinião de Assaf e Senart (2012), acaba se tornando um grande desafio.

Nas pesquisas, a definição de qualidade dos dados é frequentemente descrita como dados que são "aptos para o uso", que baseia no julgamento subjetivo da qualidade dos dados por usuários.

Para Wang e Strong (1996), um dado com qualidade é aquele que o usuário seja capaz de interpretar, seja acessível, além de relevante, exato e vir de fonte confiável.

Todavia, a qualidade dos dados é mais do que simplesmente sua exatidão, pode significar integridade, confiabilidade, consistência e atualidade. Desta forma, autores definem diferentes dimensões para caracterizar tal aspecto qualitativo.

Os autores Strong, Lee e Wang (1997) identificaram quinze dimensões es- 
senciais para a qualidade dos dados, tais como: acuracidade, objetividade, confiabilidade, reputação, acessibilidade, segurança no acesso, relevância, valor agregado, atualidade, quantidade de dados, integridade, interpretabilidade, facilidade de entendimento, representação concisa, e representação consistente.

Palvia (1996) classificou a qualidade em precisão, adequabilidade, clareza, pertinência, tempo de resposta, atualidade e utilidade de saída. Já Wand e Wang (1996) representaram as dimensões qualitativas como completude, correção, significância e inequivocidade.

Além dos autores citados, Arouck (2011) identificou mais 33 (trinta e três) autores que definiram critérios de qualidade, dos quais pode-se destacar 113 (cento e treze) termos possivelmente aplicados em sistemas de informação, tendo especial destaque: precisão, tempestividade e completude, repetidos com maior frequência.

Neste contexto, qualidade dos dados tem sido um conceito estudado e aplicado em vários campos, dentre os quais a saúde.

O Brasil dispõe de uma ampla rede de Sistemas de Informação em Saúde (SIS) de contexto nacional, com a maioria de suas informações disponível na Internet (Departamento de Informática do SUS (DATASUS).

O DATASUS surgiu em 1991, com a criação da Fundação Nacional de Saúde, e já desenvolveu mais de 200 (duzentos) sistemas que auxiliam diretamente o Ministério da Saúde no processo de construção e fortalecimento do Sistema Único de Saúde (SUS), tais como: Sistema de Informações de Nascidos Vivos (SINASC), que visa reunir informações epidemiológicas referentes aos nascimentos informados em todo território nacional e subsidiar as intervenções relacionadas à saúde da mulher e da criança para todos os níveis do SUS; Sistema de Informações de Mortalidade (SIM), para a obtenção regular de dados sobre mortalidade no país.

A partir da criação do SIM foi possível a captação de dados sobre mortalidade, de forma abrangente, para subsidiar as diversas esferas de gestão na saúde pública. Com base nessas informações é possível realizar análises de situação, planejamento e avaliação das ações e programas na área; Sistema de Informação do Câncer do Colo do Útero (SISCOLO) e Sistema de Câncer de Mama (SISMAMA), que têm como objetivo auxiliar a estruturação do "Viva Mulher", Programa Nacional de Controle do Câncer do Colo do Útero e de Mama, que coleta e processa informações sobre identificação de pacientes e laudos de exames cito patológicos e histopatológicos, fornecendo dados para o monitoramento externo da qualidade dos exames, e, assim, orientando os gerentes estaduais do programa sobre a qualidade dos laboratórios responsáveis pela leitura dos exames no município.

As informações disponibilizadas pelo DATASUS podem servir para subsidiar análises objetivas da situação sanitária, tomadas de decisão e elaboração de programas de ações de saúde. Neste sentido, a garantia da qualidade dos dados é condição essencial para a análise objetiva da situação sanitária, assim como para a tomada de decisões e para a programação de ações de saúde.

Diante do exposto, o objetivo do trabalho é realizar uma análise e discussão crítica da literatura sobre qualidade de dados do DATASUS. Para tanto, serão consideradas as publicações nas quais abordaram questões de qualidade sobre os sistemas de informação do DATASUS do ano de 1991 a 2018. Dessa forma, permitir uma melhor compreensão do que pode ser pensado como qualidade no DATASUS e que estudos poderiam ser feitos para melhorar o seu conhecimento.

\section{PROCEDIMENTOS METODOLÓ- GICOS}

A metodologia deste trabalho constitui-se de uma pesquisa exploratória com abordagem qualitativa e quantitativa, com o objetivo de identificar e analisar trabalhos que têm abordado questões de qualidade em sistemas de informação do DATASUS. Para a análise das publicações, optou-se pelas bases de dados Medline e Lilacs, por serem específicas da área da saúde. A pesquisa con- 
siderou o tema principal desse trabalho: estudos que abordaram questões de qualidade em sistemas de informação do DATASUS.

Para o levantamento dos trabalhos nas bases de dados Medline e Lilacs, realizou-se uma busca pelos termos "estatísticas vitais" e "sistemas de informação" e "qualidade de dados", considerando o ano de 1991 para início do levantamento bibliográfico, data de implantação do DATASUS, perdurando até o ano de 2018.

No cômputo do total de estudos identificados, foi verificada a duplicação entre as bases de dados, sendo cada artigo contabilizado somente uma vez. A partir dos estudos identificados, considerando a leitura dos títulos e resumos, foram selecionados os artigos que abordaram questões de avaliação de algum sistema do DATASUS.

Os artigos selecionados foram avaliados considerando a leitura e a análise criteriosa do texto completo. Após esta ação, foram excluídos aqueles claramente não re- lacionados com o tema (que abordavam a avaliação da qualidade de outros sistemas de informação, os de revisão ou tese e aqueles sem a utilização de indicadores de qualidade).

Os dados dos estudos selecionados foram extraídos de acordo com uma forma pré-definida: autor, ano, título, revista, parâmetro de avaliação e sistema avaliado, apresentados no Quadro 1.

\section{RESULTADOS}

As buscas foram realizadas por meio da aplicação da sequência de consulta para cada uma das fontes, conforme a estratégia de busca definida na Seção 2, as quais foram: "estatísticas vitais" e "sistemas de informação" e "qualidade de dados". Deste modo, foram recuperados 22 (vinte e dois) trabalhos, a maior parte dos estudos provenientes da base de dados Lilacs, com $64 \%$ dos trabalhos e $36 \%$ da base de dados Medline.

Quadro 1 - Seleção dos artigos sobre qualidade dos dados no DATASUS

\begin{tabular}{|c|c|c|c|c|c|}
\hline ID & Autor & Título & Revista & $\begin{array}{l}\text { Parâmetro de } \\
\text { avaliação }\end{array}$ & $\begin{array}{l}\text { Sistema } \\
\text { avaliado }\end{array}$ \\
\hline E01 & $\begin{array}{l}\text { Rodrigues et } \\
\text { al, } 2014\end{array}$ & $\begin{array}{l}\text { Análise espacial da mortalidade infantil e } \\
\text { adequação das informações vitais: uma } \\
\text { proposta para definição de áreas prioritá- } \\
\text { rias. }\end{array}$ & $\begin{array}{l}\text { Ciência e } \\
\text { Saúde cole- } \\
\text { tiva }\end{array}$ & $\begin{array}{l}\text { Cobertura, } \\
\text { Acurácia }\end{array}$ & $\begin{array}{l}\text { SINASC } \\
\text { e SIM }\end{array}$ \\
\hline $\mathrm{E} 02$ & $\begin{array}{l}\text { Silva et al, } \\
2012\end{array}$ & $\begin{array}{l}\text { Avaliação da qualidade dos dados do } \\
\text { Sistema de Informações sobre Nascidos } \\
\text { Vivos e do Sistema de Informações sobre } \\
\text { Mortalidade no período neonatal, Espíri- } \\
\text { to Santo, Brasil, de } 2007 \text { a } 2009 \text {. }\end{array}$ & $\begin{array}{l}\text { Ciência e } \\
\text { Saúde cole- } \\
\text { tiva }\end{array}$ & $\begin{array}{l}\text { Acessibilidade, } \\
\text { oportunidade } \\
\text { e completude }\end{array}$ & $\begin{array}{l}\text { SINASC } \\
\text { e SIM }\end{array}$ \\
\hline E03 & $\begin{array}{l}\text { Jorge et al, } \\
2007\end{array}$ & $\begin{array}{l}\text { Análise da qualidade das estatísticas vitais } \\
\text { brasileiras: a experiência de implantação } \\
\text { do SIM e do SINASC. }\end{array}$ & $\begin{array}{l}\text { Ciência e } \\
\text { Saúde cole- } \\
\text { tiva }\end{array}$ & $\begin{array}{l}\text { Oportunidade, } \\
\text { cobertura e } \\
\text { acurácia }\end{array}$ & $\begin{array}{l}\text { SINASC } \\
\text { e SIM }\end{array}$ \\
\hline E04 & $\begin{array}{ll}\text { Ferreira } & \text { et } \\
\text { al, } 2011 & \end{array}$ & $\begin{array}{l}\text { Avaliação da qualidade da informação: } \\
\text { linkage entre SIM e SINASC em Jaboatão } \\
\text { dos Guararapes (PE). }\end{array}$ & $\begin{array}{l}\text { Ciência e } \\
\text { Saúde cole- } \\
\text { tiva }\end{array}$ & $\begin{array}{l}\text { Oportunidade } \\
\text { e cobertura }\end{array}$ & $\begin{array}{l}\text { SINASC } \\
\text { e SIM }\end{array}$ \\
\hline E05 & $\begin{array}{l}\text { Rocha et al, } \\
2011\end{array}$ & $\begin{array}{l}\text { Aplicação da técnica de linkage entre as } \\
\text { bases de dados de nascidos vivos e óbitos } \\
\text { para estudo da mortalidade neonatal no } \\
\text { Recife (PE), Brasil / }\end{array}$ & $\begin{array}{l}\text { Espaço para } \\
\text { Saúde }\end{array}$ & Completude & $\begin{array}{l}\text { SINASC } \\
\text { e SIM }\end{array}$ \\
\hline E06 & $\begin{array}{l}\text { Mascarenhas } \\
\text { et al, } 2011\end{array}$ & $\begin{array}{l}\text { Confiabilidade dos dados do Sistema de } \\
\text { Informações sobre Nascidos Vivos em } \\
\text { Teresina, Estado do Piauí, Brasil - } 2002 \text { / }\end{array}$ & $\begin{array}{l}\text { Ciência e } \\
\text { Saúde cole- } \\
\text { tiva }\end{array}$ & Completude & SINASC \\
\hline E07 & $\begin{array}{l}\text { Silva et al, } \\
2001\end{array}$ & $\begin{array}{l}\text { Avaliação da qualidade dos dados do } \\
\text { sistema de informações sobre nascidos } \\
\text { vivos em } 1997-1998\end{array}$ & $\begin{array}{l}\text { Revista } \\
\text { saúde públi- } \\
\text { ca }\end{array}$ & Confiabilidade & SINASC \\
\hline
\end{tabular}

Fonte: Autora (2018). 
Após a finalização das buscas por estudos, foram realizados os processos de seleção preliminar e final dos trabalhos. Nesta etapa foram lidos os títulos e resumos de todos os textos obtidos na etapa anterior; dentre os 22 (vinte e dois) trabalhos recuperados, apenas sete abordaram informações sobre qualidade em algum sistema do DATASUS, o que representa $32 \%$ dos artigos recuperados. O resultado da seleção do número de estudos incluídos encontra-se mostrado no Quadro 1 com a distribuição quanto aos parâmetros adotados.

De acordo com os parâmetros de avaliação, constatou-se que o critério oportunidade apareceu em três estudos, cujo objetivo foi averiguar se a coleta e uso da informação ocorreu no momento certo. $\mathrm{O}$ critério cobertura, que indica a capacidade de compreender uma vasta gama de tópicos e completude, qualificando a informação como completa ou incompleta, também aparece em três estudos. Os critérios acurácia, acessibilidade e confiabilidade apareceram apenas uma vez nas publicações selecionadas; a acurácia refere-se à informação livre de erro; a acessibilidade visa identificar credibilidade perante $\mathrm{O}$ público-alvo da fonte e do conteúdo da informação; e a acessibilidade verifica se os dados estão disponíveis, facilmente ou rapidamente recuperáveis.

O Quadro 1, além de evidenciar os autores e seus respectivos critérios de avaliação de qualidade dos dados nos sistemas do DATASUS, verificou que a última publicação, de acordo com os critérios de busca definidos neste trabalho, foi em 2014, o que pode levar a novas oportunidades de pesquisa. A revisa Ciência e Saúde Coletiva foi a que mais se destacou em publicações, pois publica cerca de 30 (trinta) artigos por edição, num total de 12 (doze) edições no ano, o que pode ter contribuído com o resultado. E o mais interessante foi o resultado dos sistemas avaliados, segundo o qual as publicações se concentraram em avaliar os sistemas SINASC e SIM, talvez por fazerem parte dos primeiros sistemas implantados pelo DATASUS.

O quadro 2 apresenta a categorização dos artigos e o conteúdo chave que justifica a inclusão dos artigos.

\begin{tabular}{|c|c|}
\hline ID & Conteúdos chave dos estudos incluídos \\
\hline E01 & $\begin{array}{l}\text { "Estudo ecológico que objetivou analisar a relação entre o comportamento espacial da mortalidade infantil } \\
\text { e a adequação das informações vitais. Para avaliar a adequação das informações sobre nascimentos (Sinasc) } \\
\text { e óbitos (SIM) do Ministério da Saúde foi utilizado um método, já validado, que é constituído por cinco } \\
\text { indicadores calculados por município, segundo o porte populacional". Palavras-chave: Mortalidade infantil, } \\
\text { Sistemas de informação, Estatísticas vitais, Análise espacial. (p. 2047) }\end{array}$ \\
\hline E02 & $\begin{array}{l}\text { [...] O objetivo foi avaliar a qualidade dos dados sobre nascimentos e óbitos no período neonatal no Siste- } \\
\text { ma de Informações sobre Nascidos Vivos (SINASC) e no Sistema de Informações sobre Mortalidade } \\
\text { (SIM) no estado do Espírito Santo, no período de } 2007 \text { a 2009, os dados foram obtidos no endereço ele- } \\
\text { trônico do Departamento de Informática do Sistema Único de Saúde, no qual foram analisadas as seguin- } \\
\text { tes dimensões da qualidade das informações: acessibilidade, oportunidade e completude. apontam que as } \\
\text { informações são acessíveis e oportunas, com defasagem de três anos em relação ao início do estudo. A } \\
\text { completude das variáveis analisados no SINASC foram, na sua maioria, classificadas como excelente [...]. } \\
\text { Palavras-chave: Óbitos, Nascidos vivos, Sistema de informações, Gestão da qualidade. (p. 2011). }\end{array}$ \\
\hline E03 & $\begin{array}{l}\text { "[...] este artigo mostra a evolução dos Sistemas de Informações sobre Mortalidade e Nascidos Vivos, } \\
\text { desde a concepção, implantação, até sua avaliação. [...]mostram-se avaliações quantitativas e qualitativas } \\
\text { dos sistemas, feitas em níveis federal, estadual e municipal, visando medir a fidedignidade e as limitações } \\
\text { dos dados [...]. Palavras-chave: Sistemas de informações, Óbitos, Nascidos vivos. (p. 643). }\end{array}$ \\
\hline E04 & $\begin{array}{l}\text { "O objetivo do presente estudo foi investigar a notificação da declaração de nascidos vivos (DNV) em } \\
\text { residentes de Jaboatão dos Guararapes (PE), que foram a óbito com menos de um ano, através do linkage } \\
\text { entre o Sistema de Informação sobre Mortalidade (SIM) e o Sistema de Informação sobre Nascidos Vivos } \\
\text { (SINASC) em 2004, com a finalidade de avaliar a qualidade da informação" [...].Palavras-chave: Sistemas de } \\
\text { informação, Confiabilidade e validade, Nascido vivo. (p. 1241). }\end{array}$ \\
\hline
\end{tabular}

Fonte: Autora (2018). 
Quadro 2 - Categorização dos artigos

(continuação)

\begin{tabular}{|c|c|}
\hline ID & Conteúdos chave dos estudos incluídos \\
\hline E05 & $\begin{array}{l}\text { "O artigo objetivou analisar a aplicação da técnica de linkage entre as bases de dados do Sistema de Infor- } \\
\text { mações sobre Mortalidade (SIM) e do Sistema de Informações sobre Nascidos Vivos (SINASC) para o } \\
\text { estudo da mortalidade neonatal. Trata-se de um estudo de série temporal, composto pelos óbitos neonatais } \\
\text { originados do conjunto de nascidos vivos de mães residentes no Recife [...]. Palavras-chave: Sistemas de } \\
\text { Informação; Estatísticas Vitais; Mortalidade Neonatal; Saúde Pública. (p. 38). }\end{array}$ \\
\hline E06 & $\begin{array}{l}\text { "Este estudo avaliou a confiabilidade dos dados do Sistema de Informações sobre Nascidos Vivos (SI- } \\
\text { NASC) em Teresina, Piauí, no ano de } 2002 \text {. Estudo a proporção de preenchimento e a concordância entre } \\
\text { as variáveis do SINASC e dos registros hospitalares (SINASC-P), utilizando-se o índice kappa. Palavras- } \\
\text { chave: Nascido vivo, Declaração de nascimento, Sistemas de informação em saúde. (p. 1233). }\end{array}$ \\
\hline E07 & $\begin{array}{l}\text { "O trabalho verificou a concordância entre as informações constantes no Sistema de Informação sobre } \\
\text { Nascidos Vivos (SINASC) referentes a partos hospitalares e aquelas obtidas por inquérito seccional (pa- } \\
\text { drão-ouro). Realizou-se inquérito por amostragem em } 2.831 \text { partos hospitalares ocorridos em dez materni- } \\
\text { dades do município de São Luís, MA, abrangendo cerca de } 98 \% \text { dos nascimentos hospitalares da cidade. } \\
\text { Foram analisados: a cobertura estimada do SINASC [...]o percentual de informação ignorada ou não pre- } \\
\text { enchida e o grau de concordância entre as informações constantes no arquivo do Sinasc com o arquivo } \\
\text { perinatal. Palavras-chave: Estatísticas vitais; Sistemas de informação; Natalidade; Registro de estatísticas } \\
\text { vitais; Recém-nascido de baixo peso. Prematuro. (p. 508). }\end{array}$ \\
\hline
\end{tabular}

Fonte: Autora (2018).

A análise dos textos selecionados (Quadro 2) se deu pelas informações do resumo e das palavras-chave, pode ser que questões mais profundas do conteúdo das publicações selecionadas, que estivessem no decorrer do texto, não tenham ficado aparentes.

\section{CONSIDERAÇÕES FINAIS}

A implantação dos sistemas de saúde no DATASUS facilitou a disponibilização e o acesso a dados para análise, avaliação e gestão em saúde. Neste cenário, este trabalho buscou identificar o que tem sido tratado com relação a qualidade dos dados nos sistemas do DATASUS. Era esperado que houvesse um crescimento nos períodos mais recentes das publicações voltadas para a avaliação da qualidade de dados para esses sistemas e que houvesse estudos direcionados também para outros sistemas, tais como SISCOLO e SISMAMA.

Do total dos estudos analisados, a identificação dos parâmetros de avaliação, abordou apenas $6 \%$ dos critérios de avaliação identificados pelo estudo de Arouck (2011), o que possibilita maiores estudos em relação à qualidade dos dados dos sistemas do DATASUS.

Assim, estudos utilizando os sistemas de informação são ferramentas favoráveis para a gestão em saúde, permitindo uma visão ampliada dos agravos e possibilitando a execução de ações planejadas de acordo com características do grupo. Entretanto, é necessário o desenvolvimento de mais estudos que tenham por objetivo avaliar a qualidade dos sistemas do DATASUS, como estratégia de melhoria dos sistemas para melhor utilizálos.

\title{
DATA QUALITY OF DATASUS INFORMATION SYSTEMS: Critical analysis of literature
}

\begin{abstract}
Obtaining accurate data with quality assurance is a key factor for analysis and better decision-making in all areas. The health area provides data through the DATASUS system, which integrates several systems related to health issues. However, data quality assurance is an essential condition for objective analysis of health situation, as well as for decision-making and programming of health actions. The assignment consists of an exploratory research with a qualitative and quantitative approach, whose objective is to identify and analyze studies that have addressed quality issues in DATASUS information systems, in which a total seven selected articles that have addressed quality issues. However, it has been observed that there is still a need to develop new studies that aim to evaluate quality of DATASUS systems as an improvement strategy to better use them.
\end{abstract}




\section{Keywords}

Vital statistics. Information systems. Quality.

Artigo recebido em 11/07/2018 e aceito para publicação em 21/08/2018

\section{REFERÊNCIAS}

AROUCK, O. Atributos de qualidade da informação. 2011. 117 f. Dissertação (mestrado em Ciência da Informação) - Universidade de Brasília, Faculdade de Ciência da Informação. Brasília, 2011.

ASSAF, A.; SENART, A. Data Quality Principles in the Semantic Web. In: ICSC'12 proceedings of the 2012 IEEE Sixth International Conference on Semantic Computing. Pages 226-229 IEEE Computer Society Washington, DC, USA, 2012.

FERREIRA, A. S. J. et al. Avaliação da qualidade da informação: linkage entre SIM e SINASC em Jaboatão dos Guararapes (PE). Ciência saúde coletiva, Rio de Janeiro, v.16 supl.1. p. 1241-1246, 2011. Disponível em: http://dx.doi.org/10.1590/S141381232011000700056. Acesso em: 25 abr. 2018.

JORGE, M. H. P. M., LAURENTI R.; GOTLIEB, D. L. S. Análise da qualidade das estatísticas vitais brasileiras: a experiência de implantação do SIM e do SINASC. Ciência saúde coletiva, Rio de Janeiro, v. 12 n. 3, 2007. Disponível em http://dx.doi.org/10.1590/S141381232007000300014. Acesso em: 25 abr. 2018.

MASCARENHAS, M. D. M.; GOMES, K. R. O. Confiabilidade dos dados do Sistema de Informações sobre Nascidos Vivos em Teresina, Estado do Piauí, Brasil - 2002.

Ciência saúde coletiva, Rio de Janeiro, v. 16, suppl.1, p.1233-1239, 2011. Disponível em: http://dx.doi.org/10.1590/S1413-

81232011000700055 . Acesso em: abr. 2018.

O'BRIEN, J. A. et al. Les systemes $d$ 'information de gestion: la perspective du gestionnaire utilisateur. Quebec: Editions du Renouveau Pedagogique, 1995. 767p.

PALVIA, P. C. A model and instrument for measuring small bussiness user satisfaction with information technology. Information \& Magagement, v. 31, p. 151-163, 1996.

REDE INTERAGENCIAL DE INFORMAÇÕES PARA A SAÚDE. Indicadores básicos de saúde no Brasil: conceitos e aplicações. Brasília: Organização PanAmericana da Saúde; 2008.

ROCHA, R. et al. Aplicação da técnica de linkage entre as bases de dados de nascidos vivos e óbitos para estudo da mortalidade neonatal no Recife (PE), Brasil. Revista Espaço para a Saúde, Londrina, v. 12, n. 2, p. 38-44, jun. 2011. Disponível em: http://dx.doi.org/10.22421/1517-

7130.2011. Acesso em: 25 abr. 2018.

RODRIGUES, M. et al. Análise espacial da mortalidade infantil e adequação das informações vitais: uma proposta para definição de áreas prioritárias. Revista Ciência e Saúde Coletiva, v. 19, n.7, p. 2047 - 2054, 2014. Disponível em: http://www.scielo.br/scielo.php?pid=S1413 $81232014000702047 \&$ script $=$ sci_abstract\&tl ng=pt. Acesso em: 25 abr. 2018.

SILVA, A. A. M. et al. Avaliação da qualidade dos dados do Sistema de Informações sobre Nascidos Vivos em 1997-1998. Revista Saúde Pública, v. 35, n. 6, p. 508-514, 2001. Disponível em: http://dx.doi.org/10.1590/S003489102001000600003. Acesso em: 25 abr. 2018.

SILVA, L. P. et al. Avaliação da qualidade dos dados do Sistema de Informações sobre Nascidos Vivos e do Sistema de Informações sobre Mortalidade no período neonatal, 
Espírito Santo, Brasil, de 2007 a 2009. Ciência e saúde coletiva, v. 19, n. 7, p. 20112020, 2014. Disponível em:

http://dx.doi.org/10.1590/1413-

81232014197.08922013. Acesso em: 25 abr. 2018.

STRONG, D. M. et al. Data quality in context. Communications of the ACM, v. 40, n. 5, p. 103-110, May 1997.
WANG, R. Y.; STRONG, D. M. Beyond accuracy: what data qaulity means to data consumers. Journal of management information systems, v. 12, n.4, p. 5-34, 1996.

WANG, Y. M; WANG, Y. S. Examining the dimensionality and measurement of user perceived knowledge and information quality in the KMS contexto. Journal of Information Science, v. 35, n.1, p. 94-109, 2009. 